IPPP/06/74

DCPT/06/148

MPP-2006-139

\title{
Towards a Realistic MSSM Prediction for Neutrino-nucleon Deep-inelastic Scattering ${ }^{1}$
}

\author{
Oliver Brein*, Wolfgang Hollik ${ }^{\dagger}$ and Benjamin Koch $^{* *}$ \\ *Institute for Particle Physics Phenomenology, University of Durham, DH1 3LE, \\ Durham, United Kingdom \\ ${ }^{\dagger}$ Max-Planck-Institut für Physik, Föhringer Ring 6, D-80805 München, Germany \\ **Institut für Theoretische Physik, Johann Wolfgang Goethe-Universität, \\ D-60054 Frankfurt am Main, Germany
}

\begin{abstract}
We discuss the radiative corrections to charged and neutral current deep-inelastic neutrino-nucleon scattering in the minimal supersymmetric standard model (MSSM). In particular, we study deviations, $\delta R^{v(\bar{v})}$, from the Standard Model prediction for the ratios of neutral- to charged-current cross sections, taking into account all sources for deviations in the MSSM, i.e. different contributions from virtual Higgs bosons and virtual superpartners. Our calculation includes the full $q^{2}$ dependence of the one-loop amplitudes, parton distribution functions and a NuTeVinspired process kinematics. We present results of a scan of $\delta R^{v(\bar{v})}$ over the relevant MSSM parameter space.
\end{abstract}

\section{INTRODUCTION}

In the Standard Model (SM), neutral (NC) and charged current (CC) neutrino-nucleon scattering are described in leading order by $t$-channel $W$ and $Z$ exchange, respectively (see Fig. 1). At the NuTeV experiment, $v_{\mu}$ and $\bar{v}_{\mu}$ beams of a mean energy of $125 \mathrm{GeV}$ were scattered off a target detector and the ratios $R^{v}=\sigma_{\mathrm{NC}}^{v} / \sigma_{\mathrm{CC}}^{v}$ and $R^{\bar{v}}=\sigma_{\mathrm{NC}}^{\bar{v}} / \sigma_{\mathrm{CC}}^{\bar{v}}$ were measured. The NuTeV collaboration also provided a determination of the on-shell weak mixing angle [1], $\sin ^{2} \theta_{w}^{\text {onshell }}=0.2277 \pm 0.0013$ (stat . $) \pm 0.0009$ (syst.). This value is about $3 \sigma$ below the value derived from the residual set of precision observables [2]. The fixed-collision-energy ratios $R^{v}$ and $R^{\bar{v}}$ were not directly accessible at NuTeV. More precisely, ratios of counting rates, i.e. number of NC-like neutrino events over number of CC-like neutrino events and likewise for anti-neutrino events, $R_{\text {exp }}^{V}, R_{\text {exp }}^{\bar{v}}$, were measured and compared to SM predictions, $R_{\text {exp }}^{v}(S M), R_{\exp }^{\bar{v}}(S M)$, obtained by a detailed Monte Carlo (MC) simulation. These predictions differ from the measured values by [3]

$$
\Delta R^{v(\bar{v})}=R_{\exp }^{v(\bar{v})}-R_{\exp }^{v(\bar{v})}(S M)=-0.0032 \pm 0.0013(-0.0016 \pm 0.0028) .
$$

Basically, there are three types of explanations of the observed anomaly.

(a) There is no signal. The result might be a statistical fluctuation or theoretical errors

1 Talk given by O. Brein at SUSY06, the 14th International Conference on Supersymmetry and the Unification of Fundamental Interactions, Irvine, California, 12-17 June 2006. 

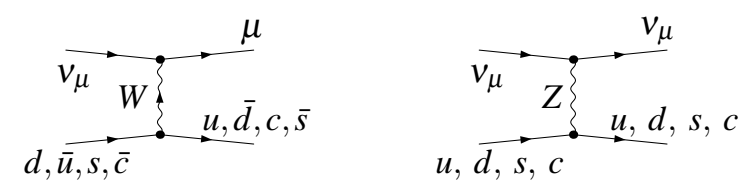

FIGURE 1. Tree-level Feynman graphs for neutral-current and charged-current scattering of muonneutrino and quark.

may have been underestimated. This line of thought lead to re-analyses of the electroweak radiative corrections to neutrino-nucleon deep-inelastic scattering (DIS)[4], which have been originally calculated almost 20 years ago [5].

(b) The apparent signal is due to neglected but relevant SM effects. An asymmetry between the distribution of the strange quark and its corresponding anti-quark in the nucleon $(s \neq \bar{s})$ could account for part of the deviation. The result of the measurement of the weak mixing angle could also be influenced by a violation of the usually assumed isospin symmetry $\left(u_{p} \neq d_{n}\right)$, or nuclear effects which have not been taken into account. For a list of other sources see Refs. [6, 7] and references therein.

(c) The signal is due to new physics. Many suggestions have been made, like effects from modified gauge boson interactions (e.g. in extra dimensions), non-renormalizable operators, leptoquarks (e.g. $R$-parity violating supersymmetry) and supersymmetric loop effects (e.g. in the MSSM), just to name a few of them (see [6, 8] and references therein for an overview).

Here we consider the effects of the radiative corrections to neutrino-nucleon DIS in the MSSM. Although the NuTeV anomaly is not settled as yet, it is interesting to check how far the MSSM could account for such a deviation. Two earlier studies [ 6, 8] treat the loop effects in the limit of zero-momentum transfer of the neutrino to the hadron, neglecting kinematical cuts and potential effects from the parton distribution functions. They conclude that the radiative corrections in the MSSM cannot be made responsible for the NuTeV anomaly, owing to the wrong sign.

Our calculation [9] includes various kinematical effects. In particular, we include the full $q^{2}$ dependence of the one-loop amplitudes, evaluate hadronic cross sections using parton distribution functions and cuts on the hadronic energy in the final state $\left(20 \mathrm{GeV}<E_{\text {had. }}<180 \mathrm{GeV}\right)$ at the NuTeV mean neutrino beam energy of $125 \mathrm{GeV}$. Moreover, we perform a thorough parameter scan for the radiative corrections $\delta R^{v(\bar{v})}$ over the relevant MSSM parameter space.

\section{MSSM RADIATIVE CORRECTIONS TO $v_{\mu} N$ DIS}

The difference between the MSSM and SM predictions, $\delta R^{n}=R_{\mathrm{MSSM}}^{n}-R_{\mathrm{SM}}^{n}$ with $R^{n}=$ $\sigma_{\mathrm{NC}}^{n} / \sigma_{\mathrm{CC}}^{n}$, with $\left(\sigma_{\mathrm{XC}}^{n}\right)_{\mathrm{NLO}}=\left(\sigma_{\mathrm{XC}}^{n}\right)_{\mathrm{LO}}+\delta \sigma_{\mathrm{XC}}^{n}(\mathrm{X}=\mathrm{N}, \mathrm{C} ; n=v, \bar{v})$, can be expanded as follows,

$$
\delta R^{n}=\left(\frac{\sigma_{\mathrm{NC}}^{n}}{\sigma_{\mathrm{CC}}^{n}}\right)_{\mathrm{LO}}\left(\frac{\left(\delta \sigma_{\mathrm{NC}}^{n}\right)_{\mathrm{MSSM}}-\left(\delta \sigma_{\mathrm{NC}}^{n}\right)_{\mathrm{SM}}}{\left(\sigma_{\mathrm{NC}}^{n}\right)_{\mathrm{LO}}}-\frac{\left(\delta \sigma_{\mathrm{CC}}^{n}\right)_{\mathrm{MSSM}}-\left(\delta \sigma_{\mathrm{CC}}^{n}\right)_{\mathrm{SM}}}{\left(\sigma_{\mathrm{CC}}^{n}\right)_{\mathrm{LO}}}\right) .
$$

Thus, only leading-order (LO) cross-sections or differences between MSSM and SM radiative corrections appear. $R$-parity conservation in the MSSM makes the Born cross section the same as in the SM (up to a negligible extra contribution involving the charged 

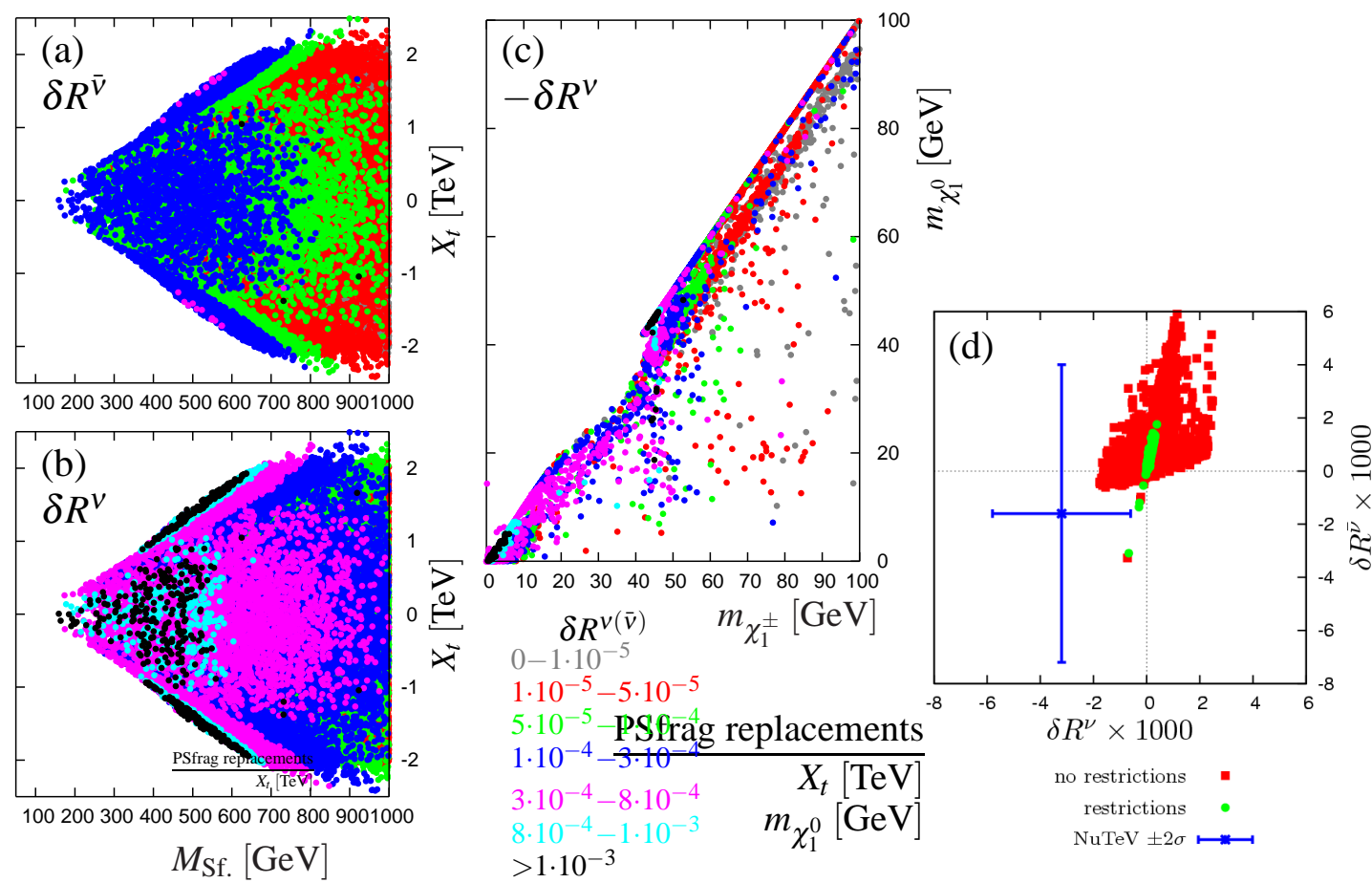

FIGURE 2. Results of parameter scan I (see text) for (a) $\delta R^{v}$ and (b) $\delta R^{\bar{v}}$ in the $M_{\text {Sf.- }}-X_{t}-$ plane. Panel

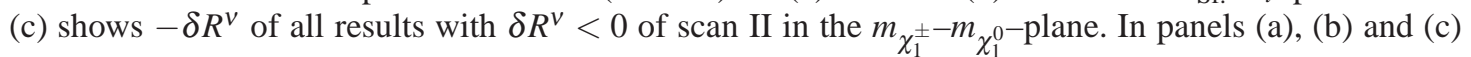
points with larger values of are plotted on top of points with smaller values. Panel (d) shows the results of scan I and II in the $\delta R^{v}-\delta R^{\bar{v}}$ plane together with the NuTeV $2 \sigma$ intervals for $\Delta R^{v}$ and $\Delta R^{\bar{v}}$.

Higgs boson). Consequently, contributions from real photon emission and all SM-like radiative corrections without virtual Higgs bosons are equal in both models. Therefore, the differences between the MSSM and SM one-loop radiative corrections boils down to the genuine superpartner (SP) loops and the difference between the Higgs-sector contributions, i.e. they are schematically given by

$$
\delta \sigma_{\mathrm{MSSM}}-\delta \sigma_{\mathrm{SM}} \propto([\mathrm{SP} \text { loops }]+[\text { Higgs graphs MSSM }- \text { Higgs graphs SM }]) .
$$

The second term in Eq. (2) vanishes when the MSSM Higgs sector is SM-like, which is the case for a $C P$-odd Higgs mass $m_{A} \gtrsim 250 \mathrm{GeV}$. The superpartner-loop contributions for CC (anti-)neutrino-quark scattering consist of $W$-boson selfenergy insertions, loop contributions to the $W q \bar{q}^{\prime}$ - and $W v_{\mu} \mu^{-}\left(W \bar{v}_{\mu} \mu^{+}\right)$-vertex, and box-graphs with doubleexchange of neutralinos and charginos. Analogous contributions appear in the NC case with $W$ replaced by $Z$ and $\mu^{-}\left(\mu^{+}\right)$by $v_{\mu}\left(\bar{v}_{\mu}\right)$. Additionally, there are SP-loop contributions to the photon- $Z$ mixing selfenergy. The difference between MSSM and SM Higgs sector contributions to the CC (NC) processes consists of the difference between all $W(Z)$ selfenergy contributions which contain at least one virtual physical Higgs boson.

\section{MSSM PARAMETER SCAN FOR $\delta R^{v(\bar{v})}$}

We perform two different parameter scans over the following region of MSSM parameter space: $10 \mathrm{GeV} \leq M_{1}, M_{2}, M_{3}, M_{\mathrm{Sf}}, m_{A^{0}} \leq 1 \mathrm{TeV}, 1 \leq \tan \beta \leq 50,-2 \mathrm{TeV} \leq$ $\mu, A_{t}, A_{b}, A_{\tau} \leq 2 \mathrm{TeV}$, where $M_{1}, M_{2}, M_{3}$ are gaugino mass parameters, $M_{\mathrm{Sf}}$. is a common 
sfermion mass scale, $\tan \beta$ is the ratio of the two Higgs vacuum expectation values in the MSSM, $\mu$ is the supersymmetric Higgs mass term, $A_{t}, A_{b}$ and $A_{\tau}$ are soft-breaking trilinear couplings. For both scans, we use the "adaptive scanning" technique [10] to scan the parameter space with an emphasis on regions where $\delta R^{v}, \delta R^{\bar{v}}$ are close to the observed deviations from the SM (Eq. 1). In scan I, we neglect all points in parameter space which violate mass exclusion limits for Higgs bosons or for superpartners or the $\Delta \rho$-constraint on sfermion mixing. Fig. 2(a) and 2(b) show the resulting values for $\delta R^{v}$ and $\delta R^{\bar{v}}$, respectively, in the $M_{\mathrm{Sf}}-X_{t}-$ plane $\left(X_{t}=A_{t}-\mu / \tan \beta\right)$. Particularly large radiative corrections occur for large stop-mixing $\left(\left|X_{t}\right| \approx 3 M_{\mathrm{Sf}}\right)$. Interestingly, the only negative values we obtain for $\delta R^{v}$ and $\delta R^{\bar{v}}$ have magnitudes below $10^{-4}$ and are not shown in Figs. 2 (a) and 2 (b). In scan II, we allow the parameter points to violate the constraints applied in scan I in order to see whether parameter regions, which could explain the $\mathrm{NuTeV}$ measurement, exist at all. Indeed, we find such points. As an example, Fig. 2(c) shows only parameter points with $\delta R^{v}<0$ resulting from scan II in the plane of the lightest neutralino and chargino mass $\left(m_{\chi_{1}^{0}}, m_{\chi_{1}^{ \pm}}\right)$, which proves to be decisive for the sign of $\delta R^{v(\bar{v})}$. Negative values with a magnitude $>10^{-3}$ only appear for $m_{\chi_{1}^{ \pm}} \lesssim 60 \mathrm{GeV}$. Thus, already the LEP-constraint on the chargino mass, $m_{\chi_{1}^{ \pm}}>94 \mathrm{GeV}$, excludes such MSSM scenarios. For heavier charginos or neutralinos than shown in Fig. 2(c) $\left|\delta R^{v}\right|$ always stays below $10^{-3}$. Fig. 2(d) relates the result of the two scans to the NuTeV measurements of $\Delta R^{v(\bar{v})}$. While scenarios with compatible values for $\delta R^{v}$ and $\delta R^{\bar{V}}$ are possible if no restrictions are taken into account, the possible predictions for $\delta R^{v}$ lie outside the $2 \sigma$ range if restrictions are applied.

\section{SUMMARY}

The NuTeV measurement of $\sin ^{2} \theta_{w}$ is intriguing but has to be further established. Especially, confirmation by other experiments is desirable. We calculated the MSSM radiative corrections to the cross section ratios $R^{v(\bar{v})}$ including several kinematic effects. It seems, MSSM loop effects do not provide a viable explanation of the deviation observed by $\mathrm{NuTeV}$. The size of the deviation could be of the right order, but it either appears with the wrong sign or violates other electroweak constraints. In any case, interesting restrictions on the MSSM parameters can be obtained. Combined with the NLO prediction in the SM, our calculation can easily provide a simulation tool for neutrino-nucleon scattering in the MSSM at the same level of accuracy.

\section{REFERENCES}

1. G. P. Zeller et al. [NuTeV Collaboration], Phys. Rev. Lett. 88 (2002) 091802. [Erratum-ibid. 90 (2003) 239902].

2. The LEP Collaborations, the LEP Electroweak Working Group and the SLD Heavy Flavor and Electroweak Working Groups, Phys. Rept. 427 (2006) 257.

3. G. P. Zeller et al. [NuTeV Collaboration], hep-ex/0207052.

4. K.-P. O. Diener, S. Dittmaier and W. Hollik, Phys. Rev. D 69 (2004) 073005, ibid. 72 (2005) 093002; A. B. Arbuzov, D. Y. Bardin and L. V. Kalinovskaya, JHEP 0506 (2005) 078.

5. D. Y. Bardin and V. A. Dokuchaeva, JINR-E2-86-260 
6. S. Davidson et al., JHEP 0202 (2002) 037.

7. S. A. Kulagin, Phys. Rev. D 67 (2003) 091301; K. S. McFarland and S. O. Moch, hep-ph/0306052; S. Kretzer et al., Phys. Rev. Lett. 93 (2004) 041802.

8. A. Kurylov, M. J. Ramsey-Musolf and S. Su, Nucl. Phys. B 667 (2003) 321.

9. O. Brein, B. Koch and W. Hollik, Proc. Internat. Conf. on Linear Colliders, LCWS 04, Paris, France, April 19-23, hep-ph/0408331.

10. O. Brein, Comput. Phys. Commun. 170, 42 (2005). 